\title{
Circular dichroism of cholesteric polymers and the orbital angular momentum of light
}

\author{
W. Löffler, ${ }^{1, \text { ⿵ D. J. Broer, }}{ }^{2}$ and J. P. Woerdman ${ }^{1}$ \\ ${ }^{1}$ Huygens Laboratory, Leiden University, P.O. Box 9504, 2300 RA Leiden, The Netherlands \\ ${ }^{2}$ Eindhoven University of Technology, Dept. Functional Organic Materials and Devices, \\ P.O. Box 513, 5600 MB Eindhoven, Netherlands
}

\begin{abstract}
We explore experimentally if the light's orbital angular momentum (OAM) interacts with chiral nematic polymer films. Specifically, we measure the circular dichroism of such a material using light beams with different OAM. We investigate the case of strongly focussed, non-paraxial light beams, where the spatial and polarization degrees of freedom are coupled. Within the experimental accuracy, we cannot find any influence of the OAM on the circular dichroism of the cholesteric polymer.
\end{abstract}

PACS numbers:

Molecular chirality is of very high importance in biology, chemistry, and material science. Materials with molecular chirality can be investigated by optical means, because chiral molecules exhibit optical activity: Their interaction with light is sensitive to the circular polarization or helicity of the photons. This interaction is enantiomerically specific, thus giving information about the structure of chiral matter (see, e.g., 1]). One manifestation of optical activity is circular dichroism, i.e., light absorption sensitive to the handedness of the circular polarization. Optical activity is thus intrinsically linked to the spin angular momentum (SAM) of the photons. More recently, it was recognized that photons can additionally carry orbital angular momentum (OAM). Such photons are naturally appearing in Laguerre-Gaussian laser modes, where each photon carries $\ell \hbar$ of OAM $(\ell$ is the azimuthal mode index [2]). Does this additional degree of freedom play a role in circular dichroism?

If such interaction would be found, it is potentially useful for a broad range of research areas and applications, comparable to (spin-based) optical activity. Along this line, the electromagnetic interaction of OAM photons with atoms and molecules has received considerable attention. However, the situation is unclear: The interaction of OAM light with atoms and molecules has been studied in a number of theoretical papers, with controversial outcomes: Some predict that such an interaction should be observable within the electric dipole approximation [3, 4], and some do not find such effects [5] 8 . The latter outcome is supported by the only experimental investigation by Araoka et al. [9]. In this experiment, the authors tested if the molecular circular dichroism (CD) of an optically active sample would be modified by using OAM probe light, with negative results. Apparently, in a molecular system, OAM does not participate in the same way in optical activity as SAM does.

Since OAM is a spatial property, it makes sense to study if OAM has an effect in a system where the main contribution to optical activity does not stem from molecular

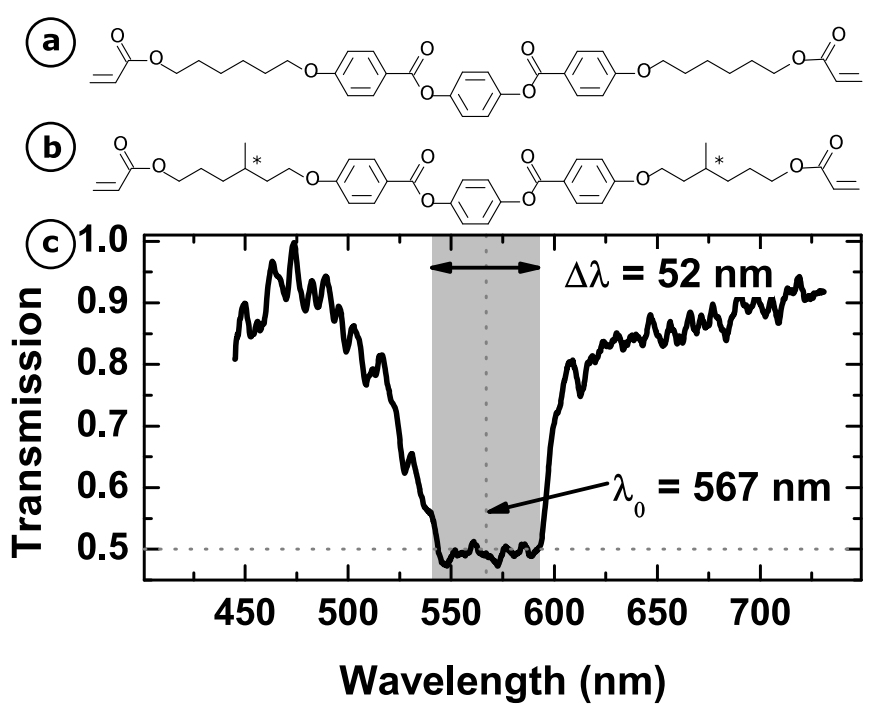

Figure 1: Structural formulas of the mesogens that we used $(a, b)$. Unpolarized transmission spectrum of the cholesteric polymer film (c). In the reflection band (gray box) the wavelength matches the pitch of the sample, there the reflected light's polarization has the same handedness as the cholesteric polymer, whereas the opposite polarization is transmitted.

chirality but from spatial resonances. Specifically, we address the structural chirality of a chiral nematic polymer, a polymerized variant of a cholesteric liquid crystal. In short-pitch chiral films, the alignment of the director has the same symmetry (helical) and periodicity (the optical wavelength) as the electric field vector in circularly polarized light (or as the wavefronts in OAM light). This results in (spin-based) optical activity effects orders of magnitude larger than in molecules. In this paper we investigate experimentally if OAM influences the circular dichroism of such a material.

We use a highly non-paraxial light beam so that the total angular momentum depends on the SAM and OAM in a non-separable way [10, this coupling emerges from spin-orbit interaction of light 11. The coupling strength 
is proportional to $\theta_{0}^{2} / 4$, where $\theta_{0}$ is the (half-) aperture angle of the focussed beam [12]. We can express the total angular momentum flux per unit length $J_{z}$, normalized to the energy flux per unit length $E$ as:

$$
\frac{J_{z}}{E}=\frac{\sigma_{z}+\ell}{\omega}+\frac{\sigma_{z}}{\omega}\left(\frac{4 / \theta_{0}^{2}}{2 p+\ell+1}+1\right)^{-1}
$$

$\sigma_{z}$ is the spin $( \pm 1), \omega$ the angular frequency, and $\ell$ and $p$ the azimuthal and radial indices of the LaguerreGaussian mode. For small NA beams, the second term is negligible as in the case of Araoka 9. We use a beam with $N A=0.55$, this results in $\theta_{0}^{2} / 4=0.1$, i.e., contributions to the total angular momentum from terms involving both SAM and OAM are significant [18.

Our cholesteric polymer films are based upon chiral nematic mixtures [13] 15] of the nematic reactive mesogen 1 and a chiral nematic reactive mesogen 2 (Fig. 1 a, b). Mesogens are monomers, which, if polymerized, show similar properties as liquid crystals. In our mixture, the wavelength of reflection can be adjusted by their mixing ratio. The materials are mixed in a 1:1 weight ratio to give reflection in the green part of the spectrum (Fig. 17). For photopolymerization, the isotropic photoinitiator Irgacure 369 (IRG369, Ciba) was added in a quantity of $1 \mathrm{w} \%$. To align the molecular layers at the bottom and top, a rubbed polyimide coating was used; to average out retardation effects, the rubbing directions were perpendicular with respect to each other. The thickness of the film was controlled by using $16 \mu \mathrm{m}$ spacers. The polymer was filled in the cell by using capillary forces and polymerized in its chiral nematic phase (at $85{ }^{\circ} \mathrm{C}$ ). Such a cholesteric polymer consists of helically oriented planes of parallel aligned molecules, and the pitch $p_{0}$ corresponds to a full $2 \pi$ rotation of the director. This results in a Bragg-type reflection band which is sensitive to the circular polarization [16, 17]: The polarization component with the same handedness as the director helix is reflected, while the other component interacts only weakly with the polymer and is transmitted. The polarization-averaged transmission spectrum (Fig. 1) shows therefore $50 \%$ transmission in the reflection band. From a nonchiral variant of the polymer, the ordinary and extraordinary refractive indices have been determined to be $n_{o} \approx 1.55$ and $n_{e} \approx 1.70$, respectively (at $\lambda=500 \mathrm{~nm}$ ). From the full width of this band $\Delta \lambda=p_{0} \Delta n$, where $\Delta n=\left|n_{e}-n_{o}\right|$, we can determine the pitch $p_{0}=347 \mathrm{~nm}$, this agrees very well with the pitch determined from the reflection band center wavelength $\lambda_{0}=\bar{n} p_{0}$ with the mean refractive index $\bar{n}=\left(n_{o}+n_{e}\right) / 2$, $p_{0}=349 \mathrm{~nm}$.

We have characterized the optical activity of our cholesteric polymer using standard diagnostics. The (spin-based) optical rotation and circular dichroism of the sample are shown in Fig. 2, Conventional solutions

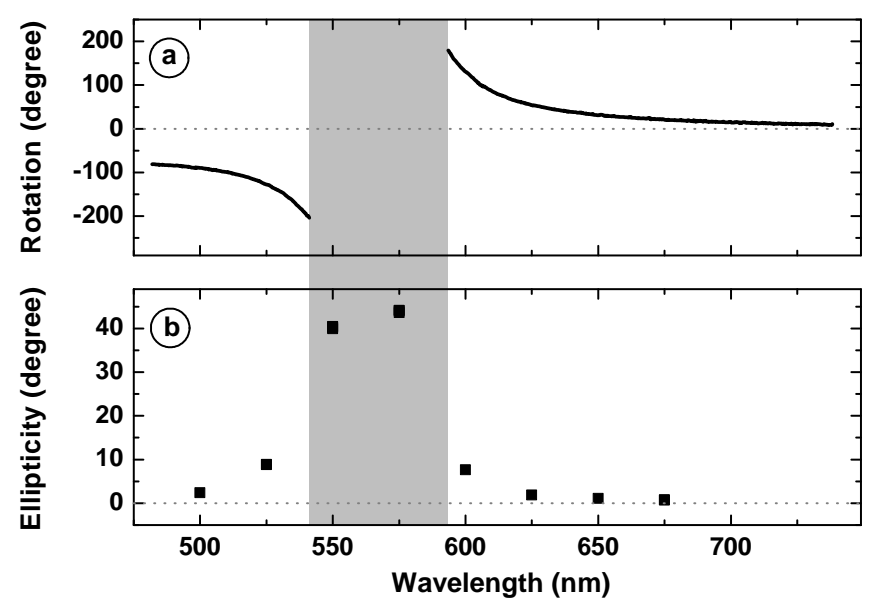

Figure 2: Measured optical rotation (a) and circular dichroism (b) of the $16 \mu \mathrm{m}$ thick cholesteric polymer. The optical rotation shows a dispersive behavior, while the $\mathrm{CD}$ that of a resonance. The $\mathrm{CD}$ is given as the ellipticity (circularly polarized light corresponds to an ellipticity of 45 degrees). The gray box indicates the reflection band from Fig. 1

of optically active molecules usually have a specific optical rotation up to a few degrees/mm. Our cholesteric polymer by far exceeds this value, close to the reflection band, the optical rotation is around $2.3 \times 10^{4}$ degrees $/ \mathrm{mm}$. In this band, the transmitted light is fully circularly polarized, and the optical rotation cannot be determined. Therefore, we have measured the circular dichroism, which can be done with high precision at any spectral position. We give in Fig. $2 \mathrm{p}$ the ellipticity $\theta$ of the transmitted light, 45 degrees corresponds to fully circularly polarized light.

For our experiment to study the effect of OAM on the circular dichroism, we use a tuneable light source a supercontinuum source (Fianium SC1060) in combination with a monochromator (full width at half maximum FWHM $=4 \mathrm{~nm}$ ). After mode-filtering the output using a singlemode fiber, we synthesize the OAM mode by holographic beam shaping with a phase-only spatial light modulator (Fig. 3) in conjunction with a spatial filter (a pinhole in the Fourier plane of a lens). The polarization of this beam is controlled with a Glan-Taylor polarizer and a photo-elastic modulator (PEM), which is set to modulate between left- and right circular polarization. The sample is at the mutual focus between two microscopy objectives (Olympus MSPlan ULWD 50x, NA $=0.55$, the backside aperture is filled by our light beam) forming a telescope. We record the transmitted light with a photo diode (PD), this signal is fed to a lock-in amplifier which is phase locked to the PEM polarization modulation $(50 \mathrm{kHz})$.

We test for a possible influence of OAM on the circular dichroism by comparing the CD signal of light with OAM $\ell=+1$ or $\ell=-1$ at various spectral positions, within 


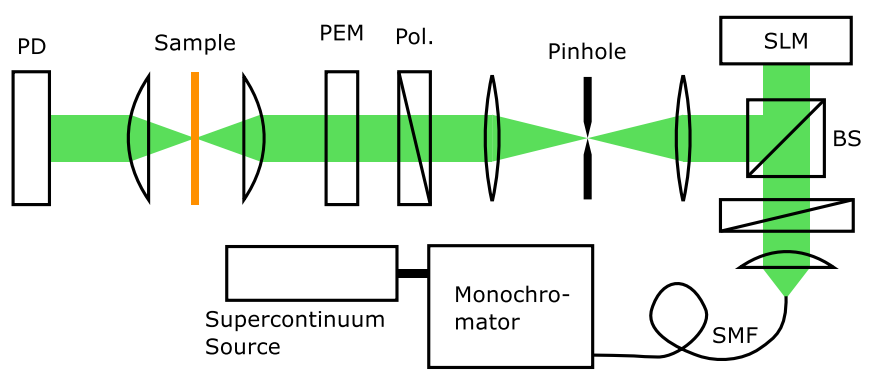

Figure 3: Scheme of the experiment. Pol.: linear polarizer; PEM: photo-elastic modulator; SLM: spatial light modulator; PD: photo diode; SMF: single-mode fiber. The PEM reference and the $\mathrm{PD}$ signal are connected to a lock-in amplifier to determine the circular dichroism.

and outside of the cholesteric reflection band. To isolate an OAM-induced effect, we introduce the normalized differential circular dichroism $\left(C D=V_{A C} / V_{D C}\right.$ is the ratio of the measured polarization-differential transmitted intensity normalized by the DC voltage):

$$
D C D=\frac{C D_{\ell=+1}-C D_{\ell=-1}}{C D_{\ell=+1}+C D_{\ell=-1}}
$$

An influence of OAM on the CD would be demonstrated if $D C D \neq 0$. Fig. 4 shows the measured DCD. We conclude that OAM has no effect here. For completeness we mention that the analogous experiment with a collimated beam, not using the microscopic telescope, shows equally a vanishing influence of OAM on the CD. We were able to determine the circular dichroism with a relative uncertainty of $0.1 \%$, this is a significant improvement compared to the experiment by Araoka et al [9], with an uncertainty of around $2 \%$.

In conclusion, we did not observe interaction of light's orbital angular momentum with a highly optically active cholesteric polymer in the non-paraxial regime. This has two implications: Firstly, OAM light does apparently not interact with the spatial resonances in a cholesteric polymer. Secondly, this demonstrates that the conversion of spin angular momentum to orbital angular momentum, as occurring at a high-NA lens, can not be reversed: A lens can not simply convert light's OAM to SAM. This agrees with recent theoretical results [11.

We acknowledge financial support by NWO and the EU STREP program 255914 (PHORBITECH).

* Electronic address: loeffler@physics.leidenuniv.nl

[1] L. D. Barron, Molecular light scattering and optical activity (Cambridge University Press, 2004).

[2] L. Allen, M. W. Beijersbergen, R. J. C. Spreeuw, and J. P. Woerdman, Phys. Rev. A, 45, 8185 (1992).

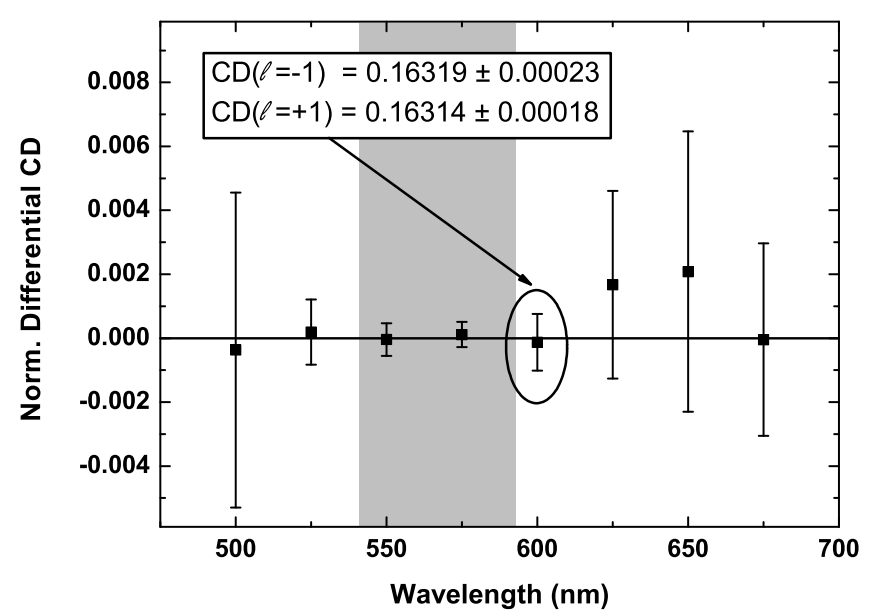

Figure 4: OAM-differential circular dichroism of the cholesteric polymer. The measurement shows the normalized difference in circular dichroism for $\ell=-1$ and $\ell=+1$ OAM beams. The probe beam was focused using a $50 \mathrm{x}, \mathrm{NA}=0.55$ objective. A non-vanishing differential CD would be an indication that OAM influences the circular dichroism. The inset shows the measured CD signal at $600 \mathrm{~nm}$ wavelength along with the uncertainty.

[3] A. Alexandrescu, D. Cojoc, and E. D. Fabrizio, Phys. Rev. Lett., 96, 243001 (2006).

[4] R. Jáuregui, Phys. Rev. A, 70, 033415 (2004).

[5] L. C. Dávila Romero, D. L. Andrews, and M. Babiker, J. Opt. B, 4, S66 (2002).

[6] D. L. Andrews, L. C. Dávila Romero, and M. Babiker, ArXiv Physics e-prints (2003), arXiv:physics/0305002.

[7] D. L. Andrews, L. C. Dávila Romero, and M. Babiker, Opt. Commun., 237, 133 (2004).

[8] M. Babiker, C. R. Bennett, D. L. Andrews, and L. C. Dávila Romero, Phys. Rev. Lett., 89, 143601 (2002).

[9] F. Araoka, T. Verbiest, K. Clays, and A. Persoons, Phys. Rev. A, 71, 055401 (2005).

[10] S. M. Barnett and L. Allen, Opt. Commun., 110, 670 (1994).

[11] K. Y. Bliokh, M. A. Alonso, E. A. Ostrovskaya, and A. Aiello, Phys. Rev. A, 82, 063825 (2010).

[12] T. A. Nieminen, A. B. Stilgoe, N. R. Heckenberg, and H. Rubinsztein-Dunlop, J. Opt. A, 10, 115005 (2008).

[13] D. J. Broer and I. Heynderickx, Macromolecules, 23, 2474 (1990).

[14] R. A. M. Hikmet, J. Lub, and D. J. Broer, Adv. Mater., 3, 392 (1991).

[15] J. Lub, D. J. Broer, R. A. M. Hikmet, and K. G. J. Nierop, Liquid Crystals, 18, 319 (1995).

[16] W. D. St. John, W. J. Fritz, Z. J. Lu, and D.-K. Yang, Phys. Rev. E, 51, 1191 (1995).

[17] D. W. Berreman and T. J. Scheffer, Phys. Rev. Lett., 25, 577 (1970).

[18] In the experiment of Araoka et al. 9] the NA of the beam was apparently much smaller than in our case. 